\title{
The Effect of Schisandrin B on the Development of Multiple Sclerosis ${ }^{\dagger}$
}

\author{
Mustafa Cakir ${ }^{1,2, *}$, Mehmet Onder Karayigit ${ }^{3}$, Ahmet Eken ${ }^{1}$ and Hamiyet Donmez-Altuntas ${ }^{1}$ \\ 1 Genome and Stem Cell Center (GENKOK), Department of Medical Biology, Faculty of Medicine, Erciyes \\ University, 38030 Kayseri, Turkey; ahmet.eken@gmail.com (A.E.); donmezh@erciyes.edu.tr (H.D.-A.) \\ 2 Department of Medical Biology, Faculty of Medicine, Yüzüncü Yıl University, 65090 Van, Turkey \\ 3 Department of Pathology, Faculty of Veterinary Medicine, Cumhuriyet University, 058140 Sivas, Turkey; \\ karayigit09@hotmail.com \\ * Correspondence: mustafacakir@erciyes.edu.tr; Tel.: +90-507-845-4901 \\ + Presented at the 3rd International conference on Natural Products for Cancer Prevention and Therapy, \\ Kayseri, Turkey, 18-20 December 2019.
}

Published: 25 December 2019

\begin{abstract}
Multiple Sclerosis (MS) is a chronic inflammatory, demyelinating neurodegenerative disease targeting the central nervous system. The pathogenesis of MS is a process of innate and adaptive immune system components. In particular, Th17 and Treg balance play an important role in the pathogenesis of MS. Several studies have shown that Th17 cells play a critical role in the pathogenesis. Schisandrin B (Sch B) is one of the most abundant and active dibenzocyclooctadiene derivatives found in the fruit of Schisandra chinensis. The aim of this study was to investigate the therapeutic effect of Sch-B and its effect on the immune cell priming in a mouse model of experimental autoimmune encephalomyelitis (EAE). Mice were immunized with MOG35-55 peptide. From day 0 , the control group received DMSO, and the Sch-B group received DMSO + Sch-B $(60$ $\mathrm{mg} / \mathrm{kg}$ ) with intraperitoneal injection every other day. On the 7th day, all mice were sacrificed and the draining lymph nodes and spleens were removed. The Foxp3 expression, Stat3 phosphorylation and the cytokines of IL-17A, IFN- $\gamma$, GM-CSF, IL-6, IL-10 and IL-22 produced from lymphocytes were analyzed by flow cytometry. In the Tlymphocytes from lymph node analyzed, IL-6 and IL-17 decreased, while IL-22 and IL-10 cytokines and Foxp3 expression increased. In the spleen T lymphocytes analyzed, Stat3 phosphorylation decreased and IL-17 cytokine increased, while IL-10 and IL-22 cytokines and Foxp3 expression increased. These results show that Sch-B decreased Th17 and increased Treg cells at the onset of EAE. In addition, EAE was scored for 18 days, Sch-B decreased the EAE clinical scores compared to the control. These results show a therapeutic effect of Sch-B in murine MS model, and warrant further studies.
\end{abstract}

Keywords: Multiple Sclerosis (MS), lymphocyte; Schisandrin B (Sch B), Th17; Treg

Acknowledgments: This work was supported by Erciyes University Scientific Research Fund (EU-BAP), Grant Number: TDK-2018-7885 (Hamiyet Donmez-Altuntas).

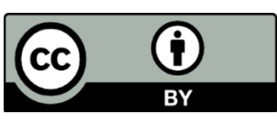

(C) 2019 by the authors. Licensee MDPI, Basel, Switzerland. This article is an open access article distributed under the terms and conditions of the Creative Commons Attribution (CC BY) license (http://creativecommons.org/licenses/by/4.0/). 